\title{
Personas sin hogar y discapacidad
}

\section{Homeless people and disability}

\section{Resumen}

Este artículo presenta los principales resultados del primer trabajo de investigación específico sobre personas sin hogar y discapacidad llevado a cabo en España. Dicho estudio se llevó a cabo en dos fases secuenciales. La Fase I perseguía cuantificar la existencia de discapacidad entre el colectivo de personas sin hogar para, en la Fase 2, profundizar en la situación de estas personas. Los resultados de la Fase I, en la que se recogió información sobre 695 personas, mostraron una importante presencia de discapacidad entre las personas sin hogar. Un I $2 \%$ de las personas sin hogar tenía certificado de discapacidad, aunque el porcentaje de discapacidad podría ser muy superior si se considera la percepción de profesionales y usuarios (al menos el $23 \%$ ). Los resultados de la Fase 2, en la que se comparaba la situación y características de las personas sin hogar con (8I participantes) y sin ella ( 48 participantes), apuntaron importantes implicaciones de la existencia de la discapacidad entre las personas sin hogar, como una mayor cronificación de la propia situación, mayores dificultades de acceso al empleo, mayor vulnerabilidad a accidentes y agresiones y otros delitos.

\section{Palabras clave}

Discapacidad, personas sin hogar, certificado de discapacidad.

\begin{abstract}
This article presents the main results of the first specific research study about homelessness and disability carried out in Spain. The study was carried out in two sequential phases: The first one (Phase I) was made in order to quantify the existence of disability among homeless people and the second one (Phase 2) was aimed at knowing the situation of these people. The results of Phase I (695 participants) showed a significant presence of disability among homeless people: I $2 \%$ of the homeless were holders of disability certificates, although the percentage of disability among people could be higher if we consider the perception of professionals and users (at least $23 \%$ ). Phase 2 compared the situation and characteristics of homeless people with (8I participants) and without ( 48 participants) disabilities. The results of the second phase showed important implications of the existence of disability among homeless, such as a greater chronicity in homelessness, more difficult access to employment and a greater vulnerability to accidents, assaults and other crimes.
\end{abstract}

\section{Key words}

Disability, homeless people, disability certificate.

\author{
Sonia Panadero Herrero \\ «spanadero@psi.ucm.es〉 \\ RAIS Fundación
}

Miguel Pérez-Lozao Gallego

‘miguel@perezlozao.es〉

RAIS Fundación

\author{
Para citar: \\ Panadero Herrero, S. y Pérez-Lozao \\ Gallego, M. (20I4): "Personas sin \\ hogar y discapacidad", Revista \\ Española de Discapacidad, 2 (2): 7-26. \\ <http://dx.doi.org/IO.5569/2340- \\ 5 IO4.02.02.0I>
}

Fecha de recepción: 1 3-07-20I4

Fecha de aceptación: 27-II-20I4 


\section{Introducción}

La existencia de una estrecha relación entre discapacidad y exclusión social, en general, ha sido puesta de manifiesto repetidamente a lo largo de los últimos años (Martínez, 20I4; Huete, 20I3).

Sin embargo, muy poco o nada se sabe sobre el papel de la discapacidad en los procesos de exclusión social asociados a ciertos colectivos como el de las personas sin hogar, uno de los colectivos más extremos dentro de estos procesos (Muñoz et al., 2003), ya que en ellos se acumulan, como probablemente en ningún otro, carencias y dificultades de muy diverso tipo -económicas, relacionales, de salud, etc.-, lo que muestra una clara vulneración de múltiples derechos reconocidos a todos los ciudadanos (Muñoz y Panadero, 2004).

Son precisamente los aspectos de salud los que han despertado quizá un mayor interés entre los investigadores. Gracias a esto actualmente se cuenta con un importante cuerpo de conocimiento sobre la vinculación entre la situación sin hogar y el deterioro de la salud de las personas que la padecen.

En general, el alojamiento inadecuado se ha asociado tradicionalmente con una pobre salud mental y física (Krieger y Higgins, 2002). Consecuentemente con esto la salud de las personas en situación sin hogar es extremadamente pobre en comparación con la población general.

Como defienden Chant et al. (20I4) en su reciente revisión sobre el tema, las personas sin hogar presentan un muy elevado número de trastornos mentales, problemas de abuso de sustancias, enfermedades crónicas e infecciosas. Concretamente, en cuanto a enfermedades físicas, las personas sin hogar sufren de forma muy frecuente: asma, trastornos cardiovasculares, cortes, problemas ginecológicos, bronquitis, gastroenteritis, diabetes, fracturas, hipertensión, etc.
Especialmente alarmante es la prevalencia de ciertas enfermedades como la tuberculosis, mucho mayores que en la población general (e.g. Feske et al., 2013; Notaro et al., 2013).

Varios estudios españoles se han interesado especialmente por la salud física de las personas sin hogar (Muñoz et al., I995; Vielva, I992; Muñoz et al., 2003), observándose importantes discrepancias entre los diferentes trabajos, debido probablemente a las diferentes metodologías utilizadas. Aún así, se puede afirmar que los datos sobre padecimiento de diferentes enfermedades resultan muy elevados. Por ejemplo, los porcentajes de tuberculosis ( $2 \%$ ) y SIDA $(6 \%)$ son en los trabajos señalados muy elevados, situándose por encima de los existentes en la población general. De forma más reciente, el Instituto Nacional de Estadística (INE, 20I2), coincidiendo con lo señalado por Cáritas (20I3), indica que casi una de cada tres personas sin hogar presenta alguna enfermedad crónica.

Este gran rango de problemas médicos probablemente podría deberse a factores diversos - pobreza, retraso en buscar asistencia sanitaria, dificultades en el acceso a los servicios de atención, no adherencia a los tratamientos, efectos de la situación sin hogar en sí misma (falta de higiene, alimentación pobre, ser víctimas de violencia), etc.- y podrían estar mediados por variables como el apoyo social (Hwang et al., 2009).

Teniendo en cuenta estos datos, sería de esperar que la mortalidad prematura entre las personas sin hogar fuera más elevada que entre la población general. Efectivamente, algunos autores han encontrado evidencia en ese sentido. Concretamente, Hwang (200I) ha señalado que entre las personas sin hogar de entre i 8 y 24 años el riesgo de fallecimiento es 5,9 veces mayor que entre la población general de esa edad, y entre los 25 y los 44 años es 3 veces mayor. Por otro lado, estudios realizados en diferentes ciudades estadounidenses hablan de una reducción drástica de la esperanza de vida media entre las personas sin hogar: la edad media de la muerte estaría entre los 4I y 47 años (Plumb, I997), siendo las enfermedades 
asociadas a mortalidad entre personas sin hogar fundamentalmente: VIH, tumores, problemas hepáticos (cirrosis), enfermedades cardiovasculares y pulmonares.

Por otro lado, la relación entre problemas de salud mental y la situación sin hogar ha sido un tema que ha despertado mucho interés a lo largo de las últimas décadas. Como indicaron ya los trabajos realizados en la década de los '90, los trastornos mentales se acumulan de una forma importante en estas personas, siendo su prevalencia superior a la de la población general e incluso a la de otros grupos económicamente desfavorecidos (Fischer y Breakey, I99I; Smith et al., I993; Scott, I993; Stein y Gelberg, I996; Farell et al., I998), situándose la prevalencia de trastornos mentales severos entre un $20 \mathrm{y}$ un 35\% (Arce et al.,I983; Koegel, Burnam, \& Farr, I988; Robertson et al., I997; Muñoz et al., I995; Toro, I998; Muñoz et al., 2003).

En España se han llevado a cabo diferentes estudios sobre salud mental entre las personas en situación sin hogar (Rico et al., I994; Lucas et al., I995; Muñoz et al., I995; Vega, I996; Muñoz et al., 2003). Aunque la metodología utilizada por cada uno de ellos les hace difícilmente comparables, se podría decir que los resultados indican una importante presencia de este tipo de trastornos. Por ejemplo, la tasa de esquizofrenia encontrada oscilaba en la mayoría de los estudios entre un 4 y un $\mathrm{I}_{3} \%$ y la de depresión mayor entre un 2 y un $20 \%$.

A la luz de estos datos, que señalan las importantes dificultades de salud que se acumulan en el colectivo de personas sin hogar, parece razonable pensar que constituyen un grupo de población con especial riesgo de sufrir una discapacidad. Sin embargo, aunque a nivel internacional se han hecho algunas aportaciones (Tsemberis et al., 2003; Herrman et al., 2004; Ho et al., 2007), los datos disponibles, que parecen apuntar efectivamente en esa dirección, son aún muy escasos.

La información existente en nuestro contexto parece indicar una cierta evidencia en este sentido. Muñoz et al. (2003) encontraron que el $22 \%$ de las personas sin hogar entrevistadas refirieron padecer una discapacidad física o sensorial, porcentaje idéntico al encontrado por Panadero (2004). En un trabajo realizado más recientemente en la ciudad de Madrid, con una metodología muy similar, este porcentaje se incrementaba hasta el $35 \%$ (Panadero y Vázquez, 20I2).

Sin embargo, son muy pocos los estudios que han analizado la vinculación entre exclusión social y discapacidad, y aún menos los que se han preguntado por la existencia de discapacidad en el colectivo de personas sin hogar de forma específica y en profundidad: no se ha documentado cómo incide la discapacidad en los procesos de exclusión de las personas sin hogar, no existen referencias empíricas o datos contrastados de las personas sin hogar que tienen además la condición de persona con discapacidad, etc.

RAIS Fundación llevó a cabo el estudio "Discapacidad en el ámbito de la exclusión social. Análisis de la situación socio-laboral y de los sistemas de protección existentes”, contratado por la Asociación FSC Discapacidad, para el Empleo y la Formación de Personas con Discapacidad, en el marco que representa la ejecución y gestión del Programa Operativo Pluriregional de "Lucha contra la Discriminación” 2007-20I3, cofinanciado por el Fondo Social Europeo (FSE), con el objetivo de realizar una aportación en ese sentido.

El objetivo general de este trabajo era analizar la posible vinculación entre exclusión y discapacidad, partiendo de un análisis de la realidad de las personas en situación de exclusión social en la Comunidad de Madrid. El objetivo de esta aproximación era profundizar en la descripción de la situación y de las experiencias de las propias personas en situación grave de exclusión social con discapacidad.

Además de la descripción de las características, situación y necesidades de las personas con discapacidad, la comparación entre estos grupos buscaba determinar las dificultades específicas de aquellas personas con discapacidad en situación 
de exclusión social frente a aquellas personas sin discapacidad en la misma situación. Pero también persigue la identificación y visibilización de aquellas personas con una probable discapacidad no reconocida oficialmente, así como sus necesidades y las barreras de acceso a los diferentes recursos de protección.

A continuación se presentan los principales resultados.

\section{Método}

El estudio sobre discapacidad en el colectivo de personas sin hogar se organizó en dos fases con objetivos y metodología diferenciadas.

La primera fase (Fase I) tenía como objetivo la cuantificación inicial de la incidencia de la discapacidad entre las personas sin hogar atendidas en los diferentes programas de RAIS Fundación en Madrid, así como una aproximación inicial a la situación de estas personas. Esta fase fue llevada a cabo entre los meses de agosto y octubre de $20 \mathrm{II}$.

La segunda fase (Fase 2) tenía como objetivo profundizar en la situación de las personas sin hogar con discapacidad respecto al colectivo de personas sin hogar en general y analizar el papel que tiene el certificado de discapacidad ${ }^{\mathrm{I}}$ para estas personas. Esta fase fue llevada a cabo a lo largo del año 2012.

\section{I. Procedimiento}

Fase 1.

En la Fase I, se solicitó a los profesionales de RAIS Fundación que cumplimentaran una ficha sobre cada uno de los usuarios en atención, a

I. Reconoce que una persona tiene discapacidad en un grado igual o superior al $33 \%$, según el procedimiento de valoración establecido por RD I97I/I999 modificado por RD I $856 / 2009$. partir de los datos recogidos en los registros de la propia entidad. En el caso de que los profesionales no contaran con la información solicitada, si era posible, se contactaba con los propios usuarios para recoger dicha información.

\section{Fase 2.}

En esta fase de profundización en la situación de las personas sin hogar con discapacidad se utilizaron diferentes estrategias (tanto cuantitativas como cualitativas) y fuentes de obtención de información (las propias personas sin hogar y profesionales de atención al colectivo). En este artículo se presentan tan sólo los principales resultados obtenidos mediante estrategias cuantitativas con los propios usuarios. Concretamente, se realizaron entrevistas estructuradas a personas sin hogar. Estas entrevistas fueron realizadas por profesionales de la propia Fundación, sin una vinculación directa con los participantes, tras recibir una sesión formativa.

Inicialmente, se entrevistó a usuarios de todos los programas de RAIS Fundación en la Comunidad de Madrid. Posteriormente, tras solicitar la colaboración del Ayuntamiento de Madrid, también fueron entrevistados usuarios de la red municipal de recursos. Concretamente, participaron usuarios del Centro de Acogida San Isidro, del Centro de Acogida Puerta Abierta y del Centro Abierto II Peñuelas.

Las entrevistas se realizaron tras recibir el consentimiento informado de los usuarios, que fueron en todos los casos informados de los propósitos del trabajo, de la voluntariedad de su participación, así como del tratamiento confidencial de los datos obtenidos durante la entrevista.

\subsubsection{Instrumentos utilizados}

Fase 1.

Como ya se ha comentado, en esta fase fueron los propios equipos profesionales los encargados de sistematizar la información sobre las personas 
usuarias en fichas individuales a partir de la información recogida en los registros de la entidad o la proporcionada por los propios usuarios. La ficha recogía información sobre los siguientes aspectos:

- $\quad$ Sexo

- Nacionalidad

- Situación de alojamiento

- Situación laboral

- Experiencia laboral

- Ingresos económicos

- Reconocimiento oficial de la discapacidad (certificado)

- Tipo de discapacidad

- Porcentaje de discapacidad

- Cuantía de los ingresos recibidos

- Acceso a recursos específicos de atención a personas con discapacidad

- Última experiencia en empleo normalizado (con contrato laboral)

- Última experiencia en empleo (sin contrato laboral)

- Percepción de la existencia de discapacidad por parte del profesional

- Percepción de la existencia de discapacidad por parte de la persona

\section{Fase 2.}

En la Fase 2 se diseñó un instrumento aplicado en forma de entrevista estructurada. Las áreas incluidas fueron las siguientes:

- Datos sociodemográficos

- Salud y discapacidad

- Situación de alojamiento

- Situación económica

- Situación laboral

- Apoyo social

- Consumo de sustancias

- Uso de recursos y barreras de acceso

- Victimización y estigma

Las preguntas incluidas fueron seleccionadas a partir de la revisión de trabajos previos tanto sobre personas sin hogar como sobre población general para posibilitar la realización de comparaciones posteriores.

\subsection{Participantes}

Fase 1.

En esta fase se recogió información sobre 695 personas en situación de exclusión social, todas ellas usuarias de RAIS Fundación en Madrid. Teniendo en cuenta la población atendida por la Fundación a lo largo del periodo en el que fue llevada a cabo esta fase, esto supuso el $65 \%$ de los usuarios atendidos en la Comunidad de Madrid a través de los diferentes programas de la Fundación (programas de Alojamiento, Piso de Apoyo al Tratamiento al Alcohol, Piso de Reinserción, Acompañamiento social del municipio de Madrid, Centro de Día “El Rincón de Magallanes", Acompañamiento social en los municipios de Móstoles, Alcobendas y San Sebastián de los Reyes).

Como se recoge en la Tabla I, en la que se presentan las principales características sociodemográficas, en su mayoría estas personas eran varones $(85,2 \%)$, españoles $(67,2 \%)$, en la mitad de los casos con edades entre 46 y 65 años.

Tabla 1. Características sociodemográficas de los participantes en la Fase 1

\begin{tabular}{|c|c|c|}
\hline & $\mathbf{n}$ & $\%$ \\
\hline \multicolumn{3}{|l|}{ Sexo } \\
\hline Hombre & 592 & 85,2 \\
\hline Mujer & 103 & 14,8 \\
\hline \multicolumn{3}{|l|}{ Edad } \\
\hline$<25$ años & 14 & 2,0 \\
\hline 25-35 años & 112 & 16,0 \\
\hline 36-45 años & 216 & 31,1 \\
\hline 46-50 años & 159 & 22,9 \\
\hline 51-65 años & 187 & 26,9 \\
\hline$<65$ años & 7 & 1,0 \\
\hline \multicolumn{3}{|l|}{ Nacionalidad } \\
\hline Española & 467 & 67,2 \\
\hline Extranjera & 228 & 32,8 \\
\hline
\end{tabular}

Fuente: Elaboración propia. 
Fase 2.

En esta segunda fase se realizaron I 29 entrevistas. Concretamente se entrevistaron 8I personas con discapacidad ( 37 de ellas con certificado de discapacidad y 44 personas que no contaban con dicho certificado) y 48 personas sin discapacidad. Para identificar el grupo de personas con discapacidad pero que carecen de certificado se consideró fundamentalmente la valoración de los propios equipos profesionales.

Las características sociodemográficas de los I 29 participantes se recogen en la tabla siguiente. Como se puede observar, estos fueron en su mayoría varones $(83 \%)$, con una media de edad de 47,57 años (DT=9,808), en la mayoría de los casos solteros $(56 \%)$.

Tabla 2. Características sociodemográficas de los participantes en la Fase 2

\begin{tabular}{|c|c|c|}
\hline & $\mathbf{n}$ & $\%$ \\
\hline \multicolumn{3}{|l|}{ Sexo } \\
\hline Varón & 107 & 82,9 \\
\hline Mujer & 22 & 17,1 \\
\hline Edad (Media (DT)) & 129 & $\begin{array}{c}46,57 \\
(9,808)\end{array}$ \\
\hline 18 a 29 años & 8 & 6,2 \\
\hline 30 a 44 años & 49 & 38,0 \\
\hline 45 a 64 años & 65 & 50,4 \\
\hline Mayores de 64 años & 7 & 5,4 \\
\hline \multicolumn{3}{|l|}{ Estado civil } \\
\hline Soltero & 72 & 55,8 \\
\hline Casado & 8 & 6,2 \\
\hline $\begin{array}{l}\text { Separado o divorciado } \\
\text { legalmente }\end{array}$ & 36 & 27,9 \\
\hline $\begin{array}{l}\text { Separado de hecho sin haber } \\
\text { iniciado ninguna tramitación } \\
\text { legal }\end{array}$ & 4 & 3,1 \\
\hline Viudo & 7 & 5,4 \\
\hline Otros & 2 & 1,6 \\
\hline \multicolumn{3}{|l|}{ Nacionalidad } \\
\hline Española & 88 & 68,2 \\
\hline Extranjera & 37 & 28,7 \\
\hline Ambas & 4 & 3,1 \\
\hline
\end{tabular}

Fuente: Elaboración propia.

\section{Análisis de datos}

Para la realización de los análisis estadísticos fue utilizado el paquete de gestión de datos SPSS versión I9.0.

A partir de los datos obtenidos se efectuaron análisis descriptivos que recogieron en primer lugar la información relativa a la muestra total de participantes.

Además, en el caso de la Fase 2, teniendo en cuenta los objetivos del presente estudio de investigación se realizaron comparaciones entre personas sin hogar con discapacidad y personas sin hogar sin discapacidad, para intentar identificar las posibles diferencias entre ambos grupos, así como entre personas con discapacidad con y sin él.

Para estas comparaciones se utilizaron, en el caso de las variables nominales, el estadístico $\chi_{2}$ (chi-cuadrado), y para variables continuas pruebas $t$ de Student para muestras independientes.

\subsection{Resultados}

\subsubsection{Resultados Fase 1}

El objetivo de esta primera fase era cuantificar la existencia de discapacidad entre las personas sin hogar. En este sentido es importante destacar que, como se recoge en la siguiente tabla, el I 2,7\% de los participantes tenía una discapacidad reconocida. En la mayoría de los casos, un 59, I \%, esta discapacidad era de tipo físico y en el $42 \%$ de los casos psíquico.

Por otro lado, en cuanto al porcentaje de discapacidad reconocida, la mitad de las personas sin hogar participantes en el estudio tenían un porcentaje reconocido de entre el 33 y el $65 \%$, aunque el $44,3 \%$ superaban este porcentaje.

Sin embargo, además de las discapacidades oficialmente reconocidas también se recogió 
Tabla 3. Participantes con discapacidad en la Fase 1 que tenían certificado de discapacidad

\begin{tabular}{|l|c|c|}
\hline & $\mathbf{n}$ & $\%$ \\
\hline $\begin{array}{l}\text { Personas con certificado de } \\
\text { discapacidad }\end{array}$ & 88 & 12,7 \\
\hline Física & 52 & 59,1 \\
\hline Psíquica & 37 & 42,0 \\
\hline Sensorial & 7 & 8,0 \\
\hline \multicolumn{3}{|l|}{ Porcentaje de discapacidad reconocida } \\
\hline$<33 \%$ & 1 & 1,1 \\
\hline $33-65 \%$ & 44 & 50,0 \\
\hline$>65 \%$ & 39 & 44,3 \\
\hline No hay datos & 4 & 4,5 \\
\hline
\end{tabular}

Fuente: Elaboración propia.

información sobre la percepción, tanto del profesional como de las propias personas entrevistadas, de la posibilidad de existencia de una discapacidad. Como se observa en la Tabla 4, los profesionales apreciaron la posible existencia de discapacidad en un $36 \%$ de los participantes, fundamentalmente de tipo psíquico $(58,4 \%)$, mientras que en el caso de los participantes este porcentaje se redujo hasta el $25 \%$ (principalmente discapacidad de tipo físico).

Por lo tanto, el acuerdo entre los profesionales y los usuarios a la hora de detectar la presencia de discapacidad no fue absoluto, aunque, como se puede observar en la tabla siguiente, los
Tabla 4. Discapacidad percibida entre los participantes en la Fase 1

\begin{tabular}{|l|c|c|c|c|}
\hline \multirow{2}{*}{} & \multicolumn{2}{|c|}{$\begin{array}{c}\text { Según el } \\
\text { profesional }\end{array}$} & \multicolumn{2}{c|}{$\begin{array}{c}\text { Según la } \\
\text { propia } \\
\text { persona }\end{array}$} \\
\cline { 2 - 5 } & $\mathbf{n}$ & $\%$ & $\mathbf{n}$ & $\%$ \\
\hline Discapacidad & 250 & 36,0 & 177 & 25,4 \\
\hline Física & 118 & 37,2 & 121 & 68,4 \\
\hline Psíquica & 185 & 58,4 & 75 & 42,4 \\
\hline Sensorial & 14 & 4,4 & 13 & 7,3 \\
\hline Sin discapacidad & 438 & 63,0 & 510 & 73,3 \\
\hline Sin datos & 7 & 1,0 & 9 & 1,3 \\
\hline
\end{tabular}

Fuente: Elaboración propia.

porcentajes de acuerdo fueron bastante elevados (por encima del azar según el índice Kappa

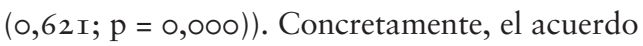
entre ambos, tanto a la hora de detectar como de descartar la existencia de discapacidad, se elevó hasta el $84 \%$.

Es decir, en el 6I \% de los casos tanto los profesionales como los usuarios descartaron la existencia de discapacidad y en el $23 \%$ de los casos ambos estaban de acuerdo con la existencia de dicha discapacidad. Sin embargo, en 20 casos los participantes señalaron tener una discapacidad que no fue detectada por los profesionales y en 93 casos ( $13,5 \%$ ) ocurrió lo contrario.

Tabla 5. Congruencia entre la percepción de las propias personas y los profesionales sobre existencia de discapacidad (Fase 1)

\begin{tabular}{|l|l|c|c|}
\hline \multicolumn{2}{|c|}{} & \multicolumn{2}{c|}{ Percepción del participante } \\
\cline { 3 - 4 } & & $\begin{array}{c}\text { No existencia de } \\
\text { discapacidad } \\
\%(\mathbf{n})\end{array}$ & $\begin{array}{c}\text { Existencia de } \\
\text { discapacidad } \\
\%(\mathbf{n})\end{array}$ \\
\hline \multirow{2}{*}{$\begin{array}{l}\text { Percepción del } \\
\text { profesional }\end{array}$} & No existencia de discapacidad & $60,7 \%(417)$ & $2,9 \%(20)$ \\
\cline { 2 - 4 } & Existencia de discapacidad & $13,5 \%(93)$ & $22,9 \%(157)$ \\
\hline
\end{tabular}

Fuente: Elaboración propia. 


\subsubsection{Resultados Fase 2}

En la Fase 2 se analizaron tanto las diferencias entre las personas sin hogar con discapacidad y sin ella, como aquellas existentes entre las primeras (con discapacidad) en función de que contaran con certificado de discapacidad o no lo hicieran (con y sin certificado).

Respecto a las características sociodemográficas de los participantes, como se puede observar en la Tabla 6, no se encontraron diferencias estadísticamente significativas en cuanto a sexo, edad, estado civil y nacionalidad entre las personas sin hogar con y sin discapacidad.

En el caso de las personas con discapacidad (Tabla 7), tampoco se encontraron diferencias estadísticamente significativas en función del sexo, edad y estado civil de los participantes en función de tener o no el certificado. Sin embargo, en este caso sí se encontraron

Tabla 6. Comparación de las características sociodemográficas de las personas sin hogar con y sin discapacidad (Fase 2)

\begin{tabular}{|c|c|c|c|c|c|}
\hline & \multicolumn{2}{|c|}{ Con discapacidad } & \multicolumn{2}{|c|}{ Sin discapacidad } & \multirow{2}{*}{$\chi^{2}$} \\
\hline & $\mathbf{n}$ & $\%$ & $\mathbf{n}$ & $\%$ & \\
\hline \multicolumn{5}{|l|}{ Sexo } & 1,121 \\
\hline Varón & 65 & 80,2 & 42 & 87,5 & \\
\hline Mujer & 16 & 19,8 & 6 & 12,5 & \\
\hline Edad (Media (DT)) & 81 & $\begin{array}{c}47,30 \\
(9,810)\end{array}$ & 48 & $\begin{array}{l}45,33 \\
(9,78)\end{array}$ & 1,375 \\
\hline \multicolumn{5}{|l|}{ Nacionalidad } & 5,052 \\
\hline Española & 61 & 75,3 & 27 & 56,3 & \\
\hline Extranjera & 18 & 22,2 & 19 & 39,6 & \\
\hline Ambas & 2 & 2,5 & 2 & 4,2 & \\
\hline
\end{tabular}

*p $\leq 0,05 ; * \mathrm{p} \leq 0,0 \mathrm{I} ; * * \mathrm{p} \leq \mathrm{0,00I}$.

Fuente: Elaboración propia.

Tabla 7. Comparación de las características sociodemográficas de las personas sin hogar con discapacidad participantes, según tengan o no certificado de discapacidad (Fase 2)

\begin{tabular}{|c|c|c|c|c|c|}
\hline & \multicolumn{2}{|c|}{ Con certificado } & \multicolumn{2}{|c|}{ Sin certificado } & \multirow{2}{*}{$\chi^{2}$} \\
\hline & $\mathbf{n}$ & $\%$ & $\mathbf{n}$ & $\%$ & \\
\hline \multicolumn{5}{|l|}{ Sexo } & 0,898 \\
\hline Varón & 28 & 75,7 & 37 & 84,1 & \\
\hline Mujer & 9 & 24,3 & 7 & 15,9 & \\
\hline Total & 37 & 100,0 & 44 & 100,0 & \\
\hline Edad (Media (DT)) & 37 & $\begin{array}{l}49,38 \\
(9,41) \\
\end{array}$ & 44 & $\begin{array}{l}45,55 \\
(9,90) \\
\end{array}$ & 1,775 \\
\hline \multicolumn{5}{|l|}{ Nacionalidad } & $9,614^{\star *}$ \\
\hline Española & 32 & 86,5 & 29 & 65,9 & \\
\hline Extranjera & 3 & 8,1 & 15 & 34,1 & \\
\hline Ambas & 2 & 5,4 & 0 & 0 & \\
\hline
\end{tabular}

*p $\leq 0,05 ; * \mathrm{p} \leq 0,0 \mathrm{I} ; * * \mathrm{p} \leq 0,00 \mathrm{I}$.

Fuente: Elaboración propia. 
diferencias estadísticamente significativas en función de la nacionalidad. Aunque en ambos casos los participantes eran en su mayoría españoles, en el caso de las personas con certificado de discapacidad este porcentaje fue significativamente mayor $(86,5 \%$ frente al $65,9 \%)$.

En la Tabla 8 se recoge la percepción de salud de los participantes con y sin discapacidad. Como resulta esperable, la percepción del estado de salud general entre los participantes con discapacidad fue significativamente peor que la de aquellas personas sin discapacidad. Mientras el $62,5 \%$ de los participantes sin discapacidad consideraba su estado de salud general bueno o muy bueno, este porcentaje se redujo hasta $30,8 \%$ entre las personas con discapacidad.

Como se observa en la Tabla 8, sí aparecieron diferencias estadísticamente significativas en el padecimiento de accidentes e intoxicaciones entre los participantes con y sin discapacidad. Un $40 \%$ de las personas sin hogar con discapacidad señaló haber sufrido algún tipo de suceso de este tipo en los I 2 meses previos a la realización de la entrevista. En el caso de las personas sin discapacidad esto se redujo hasta el I $8,8 \%$.
Estas diferencias en percepción de salud no aparecieron entre los participantes con discapacidad que tenían certificado y aquellos que no lo tenían (Tabla 9). En ambos casos la percepción de salud fue similar: la mayoría de los participantes informaron de una salud regular $(48,6 \%$ entre los que poseían el certificado y un $38,6 \%$ entre no los que no lo tenían). Además, el 21,6\% de las personas con certificado y el 29,6\% de los que carecían de él percibían su salud como mala o muy mala.

Respecto al padecimiento de accidentes, dentro del colectivo de personas con discapacidad estas diferencias desaparecen al comparar el grupo de personas con certificado y el de aquellas sin certificado. En este caso, el $35, \mathrm{x} \%$ y el $43,2 \%$ respectivamente sufrió accidentes o intoxicaciones en el año previo a la realización de la entrevista.

Según la información proporcionada por los participantes, los problemas comenzaron de media aproximadamente a los 29 años (Tabla Io). Respecto su origen, éste se había producido principalmente por enfermedades no profesionales $(33 \%)$, accidentes $(25 \%)$ y otras causas inicialmente no contempladas $(39 \%)$

\section{Tabla 8. Comparación en variables relacionadas con la salud entre las personas sin hogar con y sin} discapacidad (Fase 2)

\begin{tabular}{|c|c|c|c|c|c|}
\hline & \multicolumn{2}{|c|}{$\begin{array}{c}\text { Con } \\
\text { discapacidad }\end{array}$} & \multicolumn{2}{|c|}{$\begin{array}{c}\text { Sin } \\
\text { discapacidad }\end{array}$} & \multirow{2}{*}{$\chi^{2}$} \\
\hline & $\mathbf{n}$ & $\%$ & $\mathrm{n}$ & $\%$ & \\
\hline \multicolumn{5}{|l|}{ ¿Cuál es su estado de salud general? } & $19,997^{\star \star \star}$ \\
\hline Muy bueno & 4 & 4,9 & 12 & 25,0 & \\
\hline Bueno & 21 & 25,9 & 18 & 37,5 & \\
\hline Regular & 35 & 43,2 & 16 & 33,3 & \\
\hline Malo & 15 & 18,5 & 1 & 2,1 & \\
\hline Muy malo & 6 & 7,4 & 1 & 2,1 & \\
\hline $\begin{array}{l}\text { Durante los últimos } 12 \text { meses ¿ha tenido algún accidente } \\
\text { de cualquier tipo, incluyendo intoxicación o quemadura? }\end{array}$ & 32 & 39,5 & 9 & 18,8 & $5,989^{\star}$ \\
\hline
\end{tabular}

$* \mathrm{p} \leq 0,05 ; * \mathrm{p} \leq \mathrm{0,0 \textrm {I }} ; * * \mathrm{p} \leq \mathrm{0,00 \textrm {I }}$.

Fuente: Elaboración propia. 
Tabla 9. Comparación en variables relacionadas con la salud entre las personas sin hogar con discapacidad participantes, según tengan o no certificado de discapacidad (Fase 2)

\begin{tabular}{|l|c|c|c|c|c|}
\hline & \multicolumn{2}{|c|}{$\begin{array}{c}\text { Con } \\
\text { discapacidad }\end{array}$} & \multicolumn{2}{c|}{$\begin{array}{c}\text { Sin discapacidad } \\
\text { ¿2 }\end{array}$} \\
\cline { 2 - 6 } & $\mathbf{n}$ & $\%$ & $\mathbf{n}$ & $\%$ & 1,127 \\
\hline ¿Cuál es su estado de salud general? & 2 & 5,4 & 2 & 4,5 & \\
\hline Muy bueno & 9 & 24,3 & 12 & 27,3 & \\
\hline Bueno & 18 & 48,6 & 17 & 38,6 & \\
\hline Regular & 6 & 16,2 & 9 & 20,5 & \\
\hline Malo & 2 & 5,4 & 4 & 9,1 & \\
\hline Muy malo & & & & 19 & 43,2 \\
\hline $\begin{array}{l}\text { Durante los últimos 12 meses ¿ha tenido algún accidente } \\
\text { de cualquier tipo, incluyendo intoxicación o quemadura? }\end{array}$ & 13 & 35,1 & 19,545 \\
\hline
\end{tabular}

$* \mathrm{p} \leq 0,05 ; * \mathrm{p} \leq \mathrm{O}, \mathrm{OI} ; * * \mathrm{p} \leq 0,00 \mathrm{I}$

Fuente: Elaboración propia.

Tabla 10. Inicio de la deficiencia que dio origen a la discapacidad y pertenencia a asociaciones dedicadas a personas con discapacidad (Fase 2)

\begin{tabular}{|c|c|c|c|c|c|}
\hline & \multicolumn{2}{|c|}{$\begin{array}{l}\text { Con certificado de } \\
\text { discapacidad }\end{array}$} & \multicolumn{2}{|c|}{$\begin{array}{l}\text { Sin certificado de } \\
\text { discapacidad }\end{array}$} & \multirow[t]{2}{*}{$\chi^{2} / t^{(1)}$} \\
\hline & $\mathbf{n}$ & & $n$ & & \\
\hline $\begin{array}{l}\text { ¿Qué edad tenía al inicio de la deficiencia? } \\
\text { (Media (DT)) }\end{array}$ & 34 & $\begin{array}{c}28,62 \\
(19,175)\end{array}$ & 36 & $\begin{array}{c}29,00 \\
(17,746)\end{array}$ & $-0,011$ \\
\hline $\begin{array}{l}\text { Debido a tener una discapacidad, } \\
\text { ¿pertenece usted o sus familiares a } \\
\text { alguna asociación u organización no } \\
\text { gubernamental dedicada a personas con } \\
\text { discapacidad? }\end{array}$ & & & & & 0,309 \\
\hline No & 33 & $91,7 \%$ & 37 & $94,9 \%$ & \\
\hline Sí & 3 & $8,3 \%$ & 2 & $5,1 \%$ & \\
\hline
\end{tabular}

(I) Se ha aplicado $t$ para muestras independientes para variables continuas y pruebas de $\chi^{2}$ para las variables categóricas; *p $\leq 0,05 ; *$ p $\leq$ o, OI; *** $\leq$ o, OOI.

Fuente: Elaboración propia.

como consumo de alcohol y otras sustancias, la propia situación sin hogar o los malos tratos recibidos.

En el $45 \%$ de los casos las personas vivían en ese momento en una casa de su propiedad $(25,9 \%)$, en una vivienda de alquiler ( I I, I \%) o en alquiler compartido (8,6\%). En el 7,8\% la aparición de ésta coincidió temporalmente con la aparición de dificultades importantes relacionadas con el alojamiento.

Como se observa en la Tabla i I, las personas con discapacidad participantes en el estudio refirieron haber comenzado a sufrir problemas de alojamiento a una media de edad de 37 años, muy similar a la informada por los participantes sin discapacidad (38,56 años). 
Sin embargo, sí se encontraron diferencias estadísticamente significativas en el tiempo que los participantes de ambos grupos permanecieron en dicha situación. Mientras el grupo de personas con discapacidad permaneció más de seis años y medio en esa situación, ese tiempo se redujo hasta tres años y medio entre las personas sin hogar sin discapacidad.

\section{Respecto a la situación económica de las} personas con discapacidad participantes en el estudio, el 60,5\% de los participantes con certificado de discapacidad recibió algún tipo de ingresos relacionados con la misma, con un importe medio de 440 euros. Fundamentalmente estos ingresos fueron pensiones no contributivas (PNC) a consecuencia de la discapacidad. El 66,7\% de las personas con certificado recibió este tipo de pensiones, mientras el 23,8\% percibió pensiones de tipo contributivo. Un 9,5\% recibió otro tipo de subsidios (Tabla I2).

En el caso de los ingresos económicos totales, sí aparecieron diferencias estadísticamente significativas entre los participantes con y sin discapacidad. Las personas con discapacidad tenían unos ingresos económicos significativamente superiores ( 339 euros) a las personas sin discapacidad ( 225 euros).

\section{Tabla 11. Edad de inicio de los problemas de alojamiento y duración de la situación sin hogar (Fase 2)}

\begin{tabular}{|l|c|c|c|c|c|}
\hline & \multicolumn{2}{|c|}{$\begin{array}{c}\text { Con } \\
\text { discapacidad }\end{array}$} & \multicolumn{2}{c|}{$\begin{array}{c}\text { Sin } \\
\text { discapacidad }\end{array}$} & t \\
\cline { 2 - 6 } & $\mathbf{n}$ & & $\mathbf{n}$ & & \\
\hline ¿A qué edad tuvo por primera vez problemas & 73 & $\begin{array}{c}37,08 \\
(13,80)\end{array}$ & 48 & $\begin{array}{c}38,56 \\
(11,99)\end{array}$ & $-0,607$ \\
graves de alojamiento? Media (DT) & 71 & $\begin{array}{c}79,24 \\
(87,78)\end{array}$ & 45 & $\begin{array}{c}41,84 \\
(69,33)\end{array}$ & $2,418^{\star}$ \\
\hline $\begin{array}{l}\text { En total, sumando todos los periodos, ¿cuánto } \\
\text { tiempo se ha encontrado sin hogar? Media en } \\
\text { meses }(D T)\end{array}$ & & &
\end{tabular}

$* \mathrm{p} \leq 0,05 ; * \mathrm{p} \leq 0,0 \mathrm{I} ; * * \mathrm{p} \leq 0,00 \mathrm{I}$.

Fuente: Elaboración propia.

Tabla 12. Prestaciones económicas entre las personas sin hogar con certificado de discapacidad (Fase 2)

\begin{tabular}{|l|c|c|}
\hline \multicolumn{2}{|c|}{$\mathbf{n}$} & $\%$ \\
\hline $\begin{array}{l}\text { Como consecuencia de alguna discapacidad ¿ha recibido en los últimos 12 meses algún tipo de } \\
\text { prestación económica o indemnización? }\end{array}$ & 26 & 32,1 \\
\hline No & 49 & 60,5 \\
\hline Sí & 2 & 2,5 \\
\hline Ns/nc & \multicolumn{2}{|c|}{} \\
\hline ¿Cuáles? & 5 & $23,8^{(1)}$ \\
\hline Pensiones contributivas por incapacidad (incapacidad permanente, etc.) & 14 & $66,7^{(1)}$ \\
\hline Pensiones no contributivas a consecuencia de la discapacidad & 2 & $9,5^{(1)}$ \\
\hline Otros subsidios & 20 & 440,49 \\
\hline Importe (Media en euros (DT)) & $195,03)$ \\
\hline
\end{tabular}

(1) \% sobre aquellos que recibían algún tipo de prestación económica.

Fuente: Elaboración propia. 
Según se observa en la Tabla I3, la situación de desempleo había sido bastante prolongada en ambos grupos, no encontrándose diferencias estadísticamente significativas entre personas con y sin discapacidad. En el caso de las personas sin hogar con discapacidad hacía de media más de siete años y medio de su último trabajo con contrato, mientras en el caso de las personas sin hogar sin discapacidad esto se había prolongado durante cinco años y medio. Tampoco se encontraron diferencias significativas en cuanto a la vida laboral de los participantes en función de tener o no una discapacidad. En el caso de las personas con discapacidad el tiempo medio trabajado con contrato a lo largo de su vida era de más de ro años y medio, mientras entre las personas sin discapacidad esta media era de más de I I años y medio.

Sin embargo, sí aparecieron diferencias estadísticamente significativas entre ambos grupos en la búsqueda de empleo. Mientras el $62,5 \%$ de las personas sin discapacidad informó haber buscado empleo durante la semana previa a la entrevista, entre las personas con discapacidad este porcentaje se redujo hasta el $30,8 \%$.

Dentro de los participantes con discapacidad sí aparecieron diferencias estadísticamente significativas entre las personas con certificado y las personas que carecían de este reconocimiento oficial en ingresos económicos (Tabla I4). Así, mientras las personas con certificado recibieron durante el mes previo a la realización de la entrevista una media de 424 euros, en el caso de las personas sin certificado de discapacidad esta cifra se redujo a 267 euros.

Sin embargo, se identificaron escasas diferencias en el ámbito laboral. En primer lugar, sí aparecieron diferencias estadísticamente significativas en la realización de actividades económicas. El 23,3\% de las personas sin certificado de discapacidad había realizado algún tipo de actividad económica durante el

Tabla 13. Comparación de la situación económica y laboral entre personas con y sin discapacidad (Fase 2)

\begin{tabular}{|c|c|c|c|c|c|}
\hline & \multicolumn{2}{|c|}{ Con discapacidad } & \multicolumn{2}{|c|}{ Sin discapacidad } & \multirow{2}{*}{$\chi^{2}$} \\
\hline & $\mathbf{n}$ & & $\mathbf{n}$ & & \\
\hline $\begin{array}{l}\text { ¿Cuánto dinero recibió en total el mes pasado de } \\
\text { todas esas fuentes? Media en euros }(D T)\end{array}$ & 74 & $\begin{array}{c}339,18 \\
(265,79)\end{array}$ & 46 & $\begin{array}{l}225,46 \\
(199,12)\end{array}$ & $2,497^{*}$ \\
\hline $\begin{array}{l}\text { En el último mes, ¿ha realizado alguna actividad } \\
\text { que le haya reportado ingresos? }\end{array}$ & & & & & 0,004 \\
\hline Sí & 12 & $15,0 \%$ & 7 & $14,6 \%$ & \\
\hline No & 68 & $85,0 \%$ & 41 & $85,4 \%$ & \\
\hline $\begin{array}{l}\text { ¿Cuánto tiempo ha transcurrido desde su último } \\
\text { trabajo (con contrato)? Media en meses }(D T)\end{array}$ & 40 & $\begin{array}{c}92,33 \\
(89,90)\end{array}$ & 28 & $\begin{array}{c}67,04 \\
(68,26)\end{array}$ & 1,256 \\
\hline $\begin{array}{l}\text { ¿Durante la semana ha intentado conseguir } \\
\text { trabajo? }\end{array}$ & & & & & $12,276^{\star \star *}$ \\
\hline No & 54 & $69,2 \%$ & 18 & $37,5 \%$ & \\
\hline Sí & 24 & $30,8 \%$ & 30 & $62,5 \%$ & \\
\hline $\begin{array}{l}\text { A lo largo de su vida, y contando todos los } \\
\text { empleos que haya tenido ¿en total, durante } \\
\text { cuánto tiempo ha ejercido un trabajo con } \\
\text { contrato y/o en régimen de autónomo? Media en } \\
\text { meses }(D T)\end{array}$ & 67 & $\begin{array}{c}130,51 \\
(131,66)\end{array}$ & 47 & $\begin{array}{c}140,60 \\
(132,58)\end{array}$ & $-0,402$ \\
\hline
\end{tabular}

$* \mathrm{p} \leq 0,05 ; * \mathrm{p} \leq 0,0 \mathrm{I} ; * * \mathrm{p} \leq 0,00 \mathrm{I}$.

Fuente: Elaboración propia. 
Tabla 14. Comparación de la situación económica y laboral entre personas con discapacidad participantes, según tengan o no certificado (Fase 2)

\begin{tabular}{|c|c|c|c|c|c|}
\hline & \multicolumn{2}{|c|}{$\begin{array}{l}\text { Con certificado de } \\
\text { discapacidad }\end{array}$} & \multicolumn{2}{|c|}{$\begin{array}{l}\text { Sin certificado de } \\
\text { discapacidad }\end{array}$} & \multirow{2}{*}{$\chi^{2}$} \\
\hline & $n$ & & $n$ & & \\
\hline $\begin{array}{l}\text { ¿Cuánto dinero recibió en total el mes pasado de } \\
\text { todas esas fuentes? Media en euros }(D T)\end{array}$ & 34 & $\begin{array}{l}423,88 \\
(257,34)\end{array}$ & 40 & $\begin{array}{l}267,18 \\
(254,20)\end{array}$ & $2,628^{*}$ \\
\hline $\begin{array}{l}\text { En el último mes, ¿ha realizado alguna actividad } \\
\text { que le haya reportado ingresos? }\end{array}$ & & & & & $4,970^{*}$ \\
\hline Sí & 2 & $5,4 \%$ & 10 & $23,3 \%$ & \\
\hline No & 35 & $94,6 \%$ & 33 & $76,7 \%$ & \\
\hline $\begin{array}{l}\text { ¿Cuánto tiempo ha transcurrido desde su último } \\
\text { trabajo (con contrato)? Media en meses }(D T)\end{array}$ & 18 & $\begin{array}{c}125,11 \\
(114,85)\end{array}$ & 22 & $\begin{array}{c}65,50 \\
(51,53)\end{array}$ & 2,040 \\
\hline \multicolumn{6}{|l|}{$\begin{array}{l}\text { ¿Durante la semana ha intentado conseguir } \\
\text { trabajo? }\end{array}$} \\
\hline No & 26 & $74,3 \%$ & 28 & $65,1 \%$ & 0,762 \\
\hline Sí & 9 & $25,7 \%$ & 15 & $34,9 \%$ & \\
\hline $\begin{array}{l}\text { A lo largo de su vida, y contando todos los } \\
\text { empleos que haya tenido ¿en total, durante } \\
\text { cuánto tiempo ha ejercido un trabajo con } \\
\text { contrato y/o en régimen de autónomo? Media en } \\
\text { meses }(D T)\end{array}$ & 28 & $\begin{array}{c}116,96 \\
(113,77)\end{array}$ & 39 & $\begin{array}{c}140,23 \\
(143,80)\end{array}$ & $-0,711$ \\
\hline
\end{tabular}

$* \mathrm{p} \leq \mathrm{0,05} ; * \mathrm{p} \leq \mathrm{O}, \mathrm{OI} ; * * \mathrm{p} \leq \mathrm{O}, \mathrm{OOI}$.

Fuente: Elaboración propia.

mes previo a la realización de la entrevista, fundamentalmente de tipo informal. En el caso de las personas con certificado este porcentaje se redujo al $5,4 \%$.

Respecto al tiempo desde su último empleo, aunque las medias son bastante diferentes, estas diferencias no llegan a ser estadísticamente significativas. La media de tiempo desde el último trabajo en el caso de las personas con certificado de discapacidad supera los ro años, mientras en el caso de las personas sin este certificado este tiempo es de 5 años y medio. Tampoco se encuentran diferencias significativas en el tiempo medio trabajado con contrato o en régimen de autónomo, que es de más de 9 años y medio en el caso de las personas con certificado y de más de I I años y medio entre aquellos que no tenían certificado.

Respecto al papel que los participantes atribuían a la discapacidad en su situación (económica, laboral, familiar, etc.) en el momento de la entrevista, el 58,6\% de las personas con discapacidad percibían que ésta tenía bastante o mucho que ver con su situación, mientras el $24,3 \%$ pensaba que no había influido en ninguna medida (Tabla I 5). En este caso sí se encontraron diferencias significativas entre aquellas personas que tenían certificado de discapacidad y aquellas que no tenían dicho certificado. El grado de influencia atribuido a la discapacidad en el caso de las personas con certificado era mayor que el atribuido por las personas sin certificado. Así, mientras el 68,6\% de las personas con certificado percibía que su discapacidad había influido bastante o mucho en su situación, el porcentaje entre las personas sin certificado era del $48,5 \%$.

Respecto al uso de recursos específicos para personas con discapacidad entre los participantes, en primer lugar habría que destacar que son muy pocos los entrevistados que se encontraban siendo atendidos en algún recurso de este tipo. Como se observa en la 
Tabla 15. Percepción de causalidad de la discapacidad en la situación actual de los participantes con discapacidad (Fase 2)

\begin{tabular}{|c|c|c|c|c|c|c|c|}
\hline & \multicolumn{2}{|c|}{$\begin{array}{c}\text { Total } \\
\text { personas con } \\
\text { discapacidad }\end{array}$} & \multicolumn{2}{|c|}{$\begin{array}{c}\text { Con } \\
\text { certificado de } \\
\text { discapacidad }\end{array}$} & \multicolumn{2}{|c|}{$\begin{array}{c}\text { Sin } \\
\text { certificado de } \\
\text { discapacidad }\end{array}$} & \multirow[t]{2}{*}{$x^{2}$} \\
\hline & $n$ & $\%$ & $\mathrm{n}$ & $\%$ & $\mathbf{n}$ & $\%$ & \\
\hline $\begin{array}{l}\text { ¿Hasta qué punto cree que ha influido } \\
\text { su discapacidad en su situación actual } \\
\text { (económica, laboral, familiar, etc.)? }\end{array}$ & & & & & & & $11,214^{*}$ \\
\hline Nada & 17 & 24,3 & 10 & 28,6 & 7 & 20,0 & \\
\hline Un poco & 9 & 12,9 & 0 & 0 & 9 & 25,7 & \\
\hline Regular & 3 & 4,3 & 1 & 2,9 & 2 & 5,7 & \\
\hline Bastante & 11 & 15,7 & 7 & 20,0 & 4 & 11,4 & \\
\hline Mucho & 30 & 42,9 & 17 & 48,6 & 13 & 37,1 & \\
\hline
\end{tabular}

Fuente: Elaboración propia.

Tabla 16. Acceso a los servicios específicos de atención a personas con discapacidad (Fase 2)

\begin{tabular}{|c|c|c|c|c|c|c|c|}
\hline & \multicolumn{2}{|c|}{$\begin{array}{c}\text { Total } \\
\text { personas con } \\
\text { discapacidad }\end{array}$} & \multicolumn{2}{|c|}{$\begin{array}{c}\text { Con } \\
\text { certificado de } \\
\text { discapacidad }\end{array}$} & \multicolumn{2}{|c|}{$\begin{array}{c}\text { Sin } \\
\text { certificado de } \\
\text { discapacidad }\end{array}$} & \multirow[t]{2}{*}{$\chi^{2}$} \\
\hline & $n$ & $\%$ & $\mathbf{n}$ & $\%$ & $\mathbf{n}$ & $\%$ & \\
\hline $\begin{array}{l}\text { Actualmente, ¿utiliza o está siendo } \\
\text { atendido por algún recurso específico } \\
\text { de atención a personas con } \\
\text { discapacidad? }\end{array}$ & & & & & & & 3,130 \\
\hline Sí & 11 & 13,6 & 8 & 21,6 & 3 & 7,5 & \\
\hline No & 66 & 81,5 & 29 & 78,4 & 37 & 92,5 & \\
\hline $\mathrm{Ns} / \mathrm{nc}$ & 4 & 4,9 & & & & & \\
\hline
\end{tabular}

$* \mathrm{p} \leq 0,05 ; * \mathrm{p} \leq 0,0 \mathrm{I} ; * * \mathrm{p} \leq 0,00 \mathrm{I}$.

Fuente: Elaboración propia.

Tabla I6, el I3,6\% de las personas sin hogar con discapacidad estaba siendo atendida en estos recursos. En el caso de las personas con certificado este porcentaje se elevó hasta el 2 I, $6 \%$, mientras que en el caso de las personas sin certificado éste fue de $7,5 \%$. Sin embargo las diferencias no resultaron estadísticamente significativas.

Durante la entrevista también se recogió información sobre diferentes formas de violencia sufrida por los participantes a lo largo de su vida (Tabla I7). Además, a aquellos que habían padecido este tipo de agresiones o delitos se les preguntó si esto había ocurrido durante la situación sin hogar.

Aunque los resultados señalan una alta ocurrencia de este tipo de agresiones o delitos entre todos los participantes, como se recoge en la tabla siguiente algunas de estas situaciones fueron padecidas en mayor medida por los participantes con discapacidad. Concretamente, el 72,5\% de las personas con discapacidad había sufrido alguna agresión física a lo largo de su vida, lo que supone un porcentaje significativamente mayor que el de las personas sin discapacidad que habían sufrido este tipo de agresiones, un 43,8\%. 
Tabla 17. Comparación en padecimiento de delitos y experiencias de discriminación entre personas sin hogar participantes, según tengan o no discapacidad (Fase 2)

\begin{tabular}{|c|c|c|c|c|c|}
\hline & \multicolumn{2}{|c|}{$\begin{array}{c}\text { Con } \\
\text { discapacidad }\end{array}$} & \multicolumn{2}{|c|}{$\begin{array}{l}\text { Sin } \\
\text { discapacidad }\end{array}$} & \multirow[t]{2}{*}{$\chi^{2}$} \\
\hline & $\mathbf{n}$ & $\%$ & $\mathbf{n}$ & $\%$ & \\
\hline \multicolumn{6}{|l|}{$\begin{array}{l}\text { En algún momento de su vida, ¿ha sido víctima de } \\
\text { algún delito o agresión? }\end{array}$} \\
\hline Ha sido agredido físicamente & 58 & $72,5 \%$ & 21 & $43,8 \%$ & $10,495^{\star *}$ \\
\hline Le han robado dinero, pertenencias, documentación & 59 & $73,8 \%$ & 27 & $56,3 \%$ & $4,167^{*}$ \\
\hline Ha sufrido algún tipo de agresión sexual & 9 & $11,3 \%$ & 4 & $8,5 \%$ & 0,242 \\
\hline Le han timado & 34 & $43,0 \%$ & 19 & $41,3 \%$ & 0,036 \\
\hline Le han insultado & 62 & $77,5 \%$ & 26 & $55,3 \%$ & $6,841^{*}$ \\
\hline $\begin{array}{l}\text { En los últimos } 12 \text { meses, ¿se ha sentido discriminado } \\
\text { en alguna ocasión? }\end{array}$ & & & & & 2,942 \\
\hline Nunca & 40 & $50,0 \%$ & 27 & $56,3 \%$ & \\
\hline Algunas veces & 21 & $26,3 \%$ & 11 & $22,9 \%$ & \\
\hline Muchas veces & 15 & $18,8 \%$ & 5 & $10,4 \%$ & \\
\hline Constantemente & 4 & $5,0 \%$ & 5 & $10,4 \%$ & \\
\hline
\end{tabular}

$* \mathrm{p} \leq$ 0,05; *p $\leq$ O,OI; **p $\leq$ O,OОI.

Fuente: Elaboración propia.

También las personas sin hogar con discapacidad habían sufrido en mayor medida insultos: el 77,5\% había sufrido este tipo de violencia, porcentaje significativamente superior al $55,3 \%$ registrado entre las personas sin discapacidad.

Además, el 73,8\% de las personas con discapacidad y el $56,3 \%$ de las personas $\sin$ discapacidad habían sido víctimas de robos, diferencia estadísticamente significativa.

Para finalizar la entrevista se preguntó a los participantes si se habían sentido discriminados en alguna ocasión, durante el último año. En caso afirmativo se preguntaba a los participantes a qué atribuían dicha discriminación.

No se encontraron diferencias significativas en percepción de discriminación entre ambos grupos de participantes. El $50 \%$ de las personas con discapacidad y el $56,3 \%$ de las personas sin discapacidad no habían percibido ningún tipo de discriminación durante el último año. Sin embargo, el 23,8\% de las personas con discapacidad y el $20,8 \%$ de las personas sin discapacidad afirmaban haberse sentido discriminadas en muchas ocasiones o incluso constantemente.

\section{Conclusiones y discusión}

\subsection{La discapacidad afecta de forma muy} importante a las personas sin hogar, aunque muchas de estas personas no tienen un reconocimiento oficial de la misma

Como primera conclusión, habría que destacar que los resultados parecen confirmar las intuiciones y los escasos datos que dieron lugar al presente trabajo de investigación: la importante presencia de la discapacidad entre las personas en situación sin hogar o en situación de exclusión social severa. Así, mientras en la población general las cifras de discapacidad pueden encontrarse alrededor del 8,5\%, el 4,7\% entre las personas de entre I 6 y 64 años según 
la Encuesta sobre Discapacidades, Autonomía personal y situaciones de Dependencia (EDAD; INE, 2008), las cifras de discapacidad reconocida en población sin hogar ya superan esta cifra (el I $2 \%$ de los participantes tenía certificado de discapacidad). Esto estaría en la línea de lo encontrado por el INE en el último trabajo sobre personas sin hogar (20I2) en el que señalaron un porcentaje muy similar ( $\mathrm{I}_{5} \%$ ) y por lo recogido por Cáritas en su informe de 2013.

Pero, como ya han puesto de manifiesto diferentes autores para la población general (e.g. Pereda et al., 20I 2), la existencia de discapacidad podría ser mucho más habitual de lo que esta cifra indica, pudiendo llegar a duplicarse si se tienen en cuenta las percepciones de los propios profesionales y usuarios (altamente congruentes entre sí). Teniendo en cuenta las percepciones de ambos, existiría acuerdo en que, al menos, el $23 \%$ de los participantes presentaría algún tipo de discapacidad. Estos datos coinciden con los encontrados en los escasos trabajos que han abordado el tema hasta el momento en nuestro país (Muñoz et al., 2003; Panadero, 2004).

Aunque el tipo de discapacidad más frecuente entre los participantes fue la discapacidad física, presente en más del $70 \%$ de los casos, un papel muy relevante podrían tener también las discapacidades de tipo psíquico (especialmente mental). En la primera fase los profesionales percibían que en más del $70 \%$ de los casos las personas con discapacidad presentaban una discapacidad de tipo psíquico (en el 83,2 \% de tipo mental). Sin embargo, en la segunda fase estos resultados fueron más moderados. $\mathrm{Al}$ obtener directamente la información de las propias personas y no de los profesionales los porcentajes de discapacidades mentales fueron menores (posiblemente debido a la falta de conciencia de enfermedad que acompaña en ocasiones a estos trastornos), aunque muy elevados de todas formas: el $46 \%$ de las personas sin hogar con discapacidad tenían discapacidad de tipo mental. La discapacidad sensorial sólo afectaría a una de cada diez personas con discapacidad dentro del colectivo de personas sin hogar.
Estos datos sobre el tipo de discapacidad son muy similares a los encontrados por el INE (20I2). Según ese trabajo, a nivel nacional, el $73 \%$ de las personas sin hogar con discapacidad tendría discapacidad física, el $7 \%$ discapacidad sensorial y el $40 \%$ discapacidad psíquica.

Respecto al origen, éste se había producido principalmente por enfermedades no profesionales, accidentes y otras causas inicialmente no contempladas, como consumo de alcohol y otras sustancias, la propia situación sin hogar o los malos tratos recibidos. Estos porcentajes difieren en lo encontrado por la EDAD (INE, 2008) en población general con discapacidad. En esta encuesta se recogía que en el $60 \%$ de los casos el origen de las discapacidades se encontraba en el padecimiento de enfermedades, mientras el $9 \%$ se debía a accidentes. En este estudio los accidentes estarían en el origen del $25 \%$ de las discapacidades.

\subsection{La discapacidad es una variable relevante para los procesos de exclusión e inclusión de estas personas}

Algunos datos permiten ver el cambio que supuso para estas personas la aparición de la discapacidad en aspectos como su situación residencial o laboral. Los resultados encontrados indican que casi la mitad de las personas tenían una situación de alojamiento normalizada en el momento de aparición de la deficiencia que dio lugar a la discapacidad, por lo que en casi la mitad de los casos la situación sin hogar fue posterior a la aparición de la misma.

Resulta muy importante destacar que, a pesar de que la edad de aparición de los problemas de alojamiento fue muy similar en ambos casos, el tiempo en la situación sin hogar fue casi el doble en el caso de las personas sin hogar con discapacidad (más de 6 años y medio frente a 3 años y medio en el caso de las personas sin discapacidad), por lo que quizá la existencia de discapacidad pueda estar jugando un papel importante en la cronificación de esta situación, dificultando los procesos de inclusión de estas personas. 
Otro aspecto que se vio gravemente afectado con la aparición de la discapacidad fue el ámbito laboral. Concretamente, el $60 \%$ de las personas sin hogar entrevistadas vio modificada su relación con su actividad económica u ocupación tras la aparición de la discapacidad. Según la EDAD (INE, 2008), estos datos diferirían mucho de los encontrados en población general con discapacidad, ya que entre los participantes en esta encuesta el $80 \%$ de las personas no había sufrido cambios en su ocupación o profesión tras la aparición de la discapacidad.

Esto resulta coherente con la percepción de las propias personas sobre la influencia de su discapacidad en su situación actual. La mayoría de los participantes, aproximadamente el $60 \%$, percibía que ésta tenía bastante o mucho que ver con su situación, siendo el grado de influencia atribuido a la discapacidad en el caso de las personas con certificado mayor que el atribuido por las personas sin certificado.

\subsection{La existencia de discapacidad aumenta la ya importante vulnerabilidad de las personas sin hogar}

La discapacidad parece aumentar la vulnerabilidad ya existente en el caso de las personas sin hogar a convertirse en víctimas de agresiones y otros delitos así como a sufrir distintos tipos de accidentes.

Durante los últimos años se han llevado a cabo en España distintas investigaciones sobre el colectivo sin hogar, centradas en el estudio de los sucesos vitales estresantes padecidos por estas personas y su incidencia en la génesis y mantenimiento de su situación (Muñoz et al., I999; Muñoz et al., 2005). Estos trabajos han puesto de manifiesto que la situación sin hogar se acompaña de una gran vulnerabilidad, siendo bastante frecuente que las personas en dicha situación sean víctimas de diversos delitos. Según el estudio del INE (2005), el $40 \%$ de las personas sin hogar había sufrido algún robo desde que se encontraban en dicha situación, el $42 \%$ había padecido insultos o amenazas, el $26 \%$ había sido agredido físicamente y el 3,5\% había sufrido agresiones sexuales. Los resultados encontrados en el presente estudio apuntan también en esta dirección, ya que los datos indican una alta ocurrencia de este tipo de agresiones o delitos entre todos los participantes. Sin embargo, parece que la existencia de discapacidad hace más vulnerables aún a las personas sin hogar a este tipo de situaciones. Las personas sin hogar con discapacidad habían sufrido en mayor medida que las personas sin hogar sin discapacidad agresiones físicas, insultos y robos. La mayoría de estos delitos habían ocurrido durante la situación sin hogar, aunque no exclusivamente, es decir, estos acontecimientos no se limitaban al periodo en el que las personas se encontraban sin hogar, sino que parecían suponer una constante a lo largo de su vida.

Además, esta vulnerabilidad parece afectar también a la probabilidad de sufrir accidentes. Aproximadamente cuatro de cada diez personas sin hogar con discapacidad habían sufrido algún accidente durante el último año, el doble que las personas sin discapacidad.

Según la EDAD (INE, 2008), la discriminación percibida entre las personas con discapacidad sería un fenómeno relativamente poco frecuente: casi un $90 \%$ de las personas con discapacidad informó no haberse sentido discriminada en ninguna ocasión a lo largo del año anterior. Sin embargo, la discriminación parece ser, en alguna medida, una experiencia habitualmente vivida por las personas sin hogar. En datos del INE (2OI2) aproximadamente la mitad de las personas sin hogar se había sentido discriminada en alguna ocasión a lo largo del último año, porcentaje muy similar al encontrado en el presente estudio. Estos resultados parecen coincidir en el caso de las personas con y sin discapacidad dentro del colectivo de personas sin hogar, no encontrándose diferencias significativas entre los participantes.

\subsection{El certificado facilita el acceso a prestaciones económicas}

El contar con el reconocimiento oficial de la discapacidad parece ayudar en alguna medida a lograr el acceso a prestaciones económicas 
vinculadas a la discapacidad y supone una ventaja en cuanto al importe de las mismas, en comparación con otras prestaciones.

Diversos trabajos han abordado el tema de la situación económica de las personas con discapacidad. Pereda et al. (20I2) señalan que los ingresos de las personas con discapacidad en edad laboral eran, antes de las ayudas, un $34 \%$ inferior a la media de la población y pasaban a ser un I3 \% inferior después de las ayudas. Además, la proporción de personas del colectivo bajo el umbral de la pobreza (I9,5\%) era mucho mayor que el de las personas sin limitaciones (I 2,8 \%) (Applica; Cesep \& Alpahametrics, 2007).

En este caso, todas las personas que participaron en el estudio se encontraban en una situación de exclusión social severa y en esta situación extrema la existencia de una discapacidad oficialmente reconocida puede suponer en ocasiones una fuente de ingresos. Concretamente, en este caso el $60 \%$ de los participantes con certificado de discapacidad recibió algún tipo de ingreso relacionado con la misma, fundamentalmente pensiones no contributivas a consecuencia de la discapacidad.

Es en este punto en el que se percibe más claramente la utilidad del certificado de discapacidad. La existencia de una discapacidad reconocida suponía una ventaja desde el punto de vista económico, ya que estas personas tenían unos ingresos económicos superiores $(423$ euros de media) a las personas sin discapacidad (225 euros de media) y a las personas con discapacidad sin certificado ( 267 euros).

\subsection{Las personas sin hogar no acceden a recursos de atención a la discapacidad}

Las dificultades de acceso de las personas sin hogar a los recursos existentes, en especial a los recursos sanitarios, han sido ampliamente estudiadas (Craig y Timms, 2000; Hwang et al., 20I0). Según apuntan los resultados del presente estudio parece que estas dificultades alcanzan también a los recursos de atención a las personas con discapacidad. El $86 \%$ de los participantes con discapacidad no estaba siendo atendido en ningún recurso específico de atención a personas con discapacidad. Estas dificultades de acceso podrían estar relacionadas con características de los propios recursos (como la falta de plazas) pero también con la rigidez de los mismos en sus criterios de acceso, que no consideran la incorporación de estas personas por la acumulación de diferentes problemas: consumo de alcohol u otras sustancias, situación sin hogar, etc. También podrían estar relacionados con la imagen negativa del colectivo de personas sin hogar o la ausencia de familia (o la existencia de unas relaciones familiares deterioradas) que hacen que exista una menor presión al acceso de estas personas (Panadero y Pérez-Lozao, 20I3).

Antes de terminar resulta importante hacer también algunas consideraciones sobre la muestra del estudio. Como ya se comentó en la metodología, los participantes en este estudio eran fundamental, aunque no exclusivamente, usuarios de la RAIS Fundación, por lo que la representatividad de la misma no está garantizada. Sin embargo, es importante señalar que las dos submuestras (personas sin hogar con y sin discapacidad) resultaron equivalentes en sexo, edad, estado civil y nacionalidad, respondiendo a las características generales de la muestra y del colectivo sin hogar, es decir, mayoritariamente varones (al menos ocho de cada diez participantes), solteros, con una edad media por encima de los 45 años y en más de la mitad de los casos españoles. Esto hace difícil que las diferencias encontradas en el trabajo entre personas con y sin discapacidad pudieran atribuirse en alguna medida a estos factores.

Sin pretender olvidar sus limitaciones, este estudio ha aportado nuevos conocimientos sobre la situación de las personas sin hogar con discapacidad, ayudando así a visibilizar su difícil realidad y sus necesidades específicas, lo que supone un paso previo imprescindible para dar una respuesta adecuada y eficaz a las mismas. 
Referencias bibliográficas

Applica; Cesep \& Alpahametrics (2007): Men and women with disabilities in the EU: statistical analysis of the LFS ad hoc module and the EUSILC, Brussels: European Commission.

Arce, M. et al. (1983): “A psychiatric profile of street people admitted to an emergency shelter", Hospital and Community Psychiatry, 34: 8I2-8I7.

Cáritas (2013): La salud de las personas en situación de sin hogar acompañadas por Cáritas, Madrid: Cáritas Española.

Chant, C. et al. (2OI4): "Critical illness in homeless persons is poorly studied: a systematic review of the literature", Intensive Care Medicine, 4O(I): I 23-I 25 .

Craig, T. \& Timms, P. (2000) : "Facing up to social exclusion: services for homeless mentally ill people", International review of psychiatry, I 2(3): 206-2II.

Farell, M. et al. (1998): "Substance misuse and psychiatric comorbility: an overview of the OPCS National Psychiatric Morbility Survey”, Addictive Behaviors, 23(6): 909-1018.

Feske, M. L. et al. (2013) "Counting the Homeless: A Previously Incalculable Tuberculosis Risk and Its Social Determinants", American Journal of Public Health, I03(5): 839-848.

Fischer, P. J. \& Breakey, W. R. (I99I): “The epidemiology of alcohol, drug and mental disorders among homeless persons", American psychologist, 46(I I): I I I 5-I I 28.

Herrman, H. et al. (2004): "Disability and service use among homeless people living with psychotic disorders Australian and New Zealand”, Journal of Psychiatry, 38: 965-974.

Ho, P. et al. (2007): "Health and Housing among Low-Income Adults with Physical Disabilities", Journal of Health Care for the Poor and Underserved, I 8(4): 902-9I 5 .

Huete, A. (2013): "La exclusión de la población con discapacidad en España. Estudio específico a partir de la Encuesta Social Europea”, Revista

Española de Discapacidad, I(2): 7-24.

Hwang, S. W. et al. (2010): "Universal Health Insurance and Health Care Access for Homeless Persons", American Journal of Public Health, Iо0(8): I454-I46I.

Hwang, S. et al. (2009): "Multidimensional Social Support and the Health of Homeless Individuals", Journal of Urban Health: Bulletin of the New York Academy of Medicine, 86(5): 79I- 803 .

Hwang, S. W. (200I): "Homeless and Health", Canadian Medical Association Journal, I64(2): 229-233.

Instituto Nacional de Estadística (20I2): Encuesta a las personas sin hogar, Madrid: INE.

Instituto Nacional de Estadística (2008): Encuesta sobre Discapacidad, Autonomía personal y Situaciones de Dependencia (EDAD), Madrid: INE.

Instituto Nacional de Estadística (2005): Encuesta sobre las personas sin hogar (EPSH 2005), Madrid: INE.

Koegel, P; Burnam, A. \& Farr, R. (1988): “The prevalence of specific psychiatric disorders among homeless individuals in the inner city or Los Angeles", Archives of general psychiatry, 45(I2): I08 5-I092.

Krieger, J. \& Higgins, D (2002): "Housing and health: Time again for public health action", American Journal of Public Health, 92(5): 758-768.

Lucas, R. et al. (I995): "Prevalencia de sintomatología psicótica y hábitos tóxicos en una muestra de homeless", Revista psiquiátrica de la Facultad de Medicina de Barcelona, 22(I): I 8-24.

Martínez, B. (20I4): "Pobreza, discapacidad y derechos humanos", Revista Española de Discapacidad, I(I): 9-32. 
Muñoz, M. et al. (I999): "Stressful life events among homeless people", Journal of Community Psychology, 27(I): 73-87.

Muñoz, M. y Panadero, S (2004): "Personas sin hogar y derechos humanos: Los límites de la exclusión”. En: De la Corte, L. (eds): Psicología $y$ derechos humanos, Madrid: Editorial Icaría.

Muñoz, M. et al. (2005): "Role of stressful life events in homelessness: An Intragroup análisis", American Journal of Community Psychology, 35(I/2): 35-47.

Muñoz, M. et al. (2003): Los límites de la exclusión: Estudio sobre los factores económicos, psicosociales y de salud que afectan a las personas sin hogar en Madrid, Madrid: Témpora.

Muñoz, M. et al. (1995): Personas sin hogar en la Comunidad de Madrid. Informe psicosocial y epidemiológico, Madrid: Comunidad Autónoma de Madrid.

Notaro, K. et al. (2OI3): "Analysis of the Health Status of the Homeless Clients Utilizing a Free Clinic", Journal of Community Health, $38(\mathrm{I})$ : I72-I77.

Panadero, S. (2004): Evaluación de programas psicosociales para personas sin hogar en Madrid. Tesis doctoral, Universidad Complutense de Madrid, Facultad de Psicología.

Panadero, S. y Vázquez, J. J. (20I 2): “La Investigación sobre Personas sin Hogar y los Recursos de Atención al Colectivo en España. Evolución, Situación Actual y Retos Futuros". En: Zúñiga, C. (ed): Psicología, Sociedad y Equidad: Aportes y Desafíos, Santiago de Chile: Práxis Psicológica: 87-107.

Panadero, S. y Pérez-Lozao, M. (2013): Discapacidad en el ámbito de la exclusión social, Asociación FSC Discapacidad para el Empleo y la Formación de Personas con Discapacidad (inédito).

Pereda, C. et al. (2012): Discapacidades e inclusión social, Barcelona: Obra Social La Caixa.

Plumb, J. D. (I997): “Homelessness: Care, prevention and public policy", Annals of Internal Medicine, I 26(2): 973-75.
Rico, P. et al. (I994): “Trastornos psiquiátricos en transeúntes: un estudio epidemiológico en Aranjuez”, Revista de la Asociación Española de Neuropsiquiatría, I4: 633-649.

Robertson, M. J. et al. (I997): "Drug use disorders and treatment contact among homeless adults in Alameda County, California", American journal of public health, 87(2): 22I-228.

Scott, J. (I993): "Homeless and mental illness", British Journal of Psychiatry, I62: 314-324.

Smith, E. M. et al. (1993): “Alcohol, drugs and psychiatric comorbility among homeless women: An epidemiologic study", Journal of clinical psychiatry, 54(3): 82-87.

Stein, J. A. \& Gelberg, L. (I996): “Comparability and representativeness of clinical homeless, comunity homeless and domicilied clinic samples: physical and mental health, substance use and health services utilization", Health psychology, I6(2): I 5 5-162.

Toro, P. A. (1998): "Homeless". En: Bellock, S. \& Hersen, M. (eds): Comprehensive Clinical Psychology, Elservier Science.

Tsemberis, S. J. et al. (2003): "Consumer Preference Programs for Individuals Who Are Homeless and Have Psychiatric Disabilities", American Journal of Community Psychology, $32(3 / 4): 305$.

Uribe, J. y Alonso, S. (2010): "Personas en situación de sin hogar en Barcelona. Perfiles, estado de salud y atención sanitaria”, Revista Humanización, pastoral y ética de la salud, 295-296: I-IO8.

Vega, L. S. (I996): Salud mental en población sin hogar: Estudio epidemiológico en albergues para transeúntes de Gijón (Asturias), Oviedo: Sespa.

Vielva, C. (I992): "Estrés psicosocial y alteraciones emocionales en transeúntes marginados. Papel modular de las redes de apoyo social”, Intervención psicosocial, I: 79-86. 